\title{
Are We Predicting Disease Progress of the Rectal Cancer Patients without Surgery after Neoadjuvant Chemoradiotherapy?
}

\author{
Bo Young Oh, $\mathrm{MD}, \mathrm{PhD}$ \\ Jung Wook Huh, MD, PhD² \\ Woo Yong Lee, MD, PhD² \\ Yoon Ah Park, MD² \\ Yong Beom Cho, MD, PhD² \\ Seong Hyeon Yun, MD, PhD² \\ Hee Cheol Kim, MD, PhD² \\ Ho-Kyung Chun, MD, PhD ${ }^{3}$
}

\author{
${ }^{1}$ Department of Surgery, Ewha Womans \\ University School of Medicine, Seoul, \\ ${ }^{2}$ Department of Surgery, Samsung Medical \\ Center, Sungkyunkwan University School of \\ Medicine, Seoul, ${ }^{3}$ Department of Surgery, \\ Kangbuk Samsung Hospital, Sungkyunkwan \\ University School of Medicine, Seoul, Korea
}

Correspondence: Jung Wook Huh, MD, PhD Department of Surgery, Samsung Medical Center, Sungkyunkwan University School of Medicine, 81 Irwon-ro, Gangnam-gu, Seoul 06351, Korea

Tel: 82-2-3410-1826

Fax: 82-2-3410-6980

E-mail: jungwook.huh@gmail.com

Co-correspondence: Woo Yong Lee, MD, PhD Department of Surgery, Samsung Medical Center, Sungkyunkwan University School of Medicine, 81 Irwon-ro, Gangnam-gu, Seoul 06351, Korea

Tel: 82-2-3410-0261

Fax: 82-2-3410-6980

E-mail: 1wy555@skku.edu

Received February 8, 2017

Accepted June 21, 2017

Published Online July 3, 2017

\section{Purpose}

There are patients who do not undergo surgery, regardless of tumor response for neoadjuvant chemoradiotherapy (nCRT) in rectal cancer. However, there have been few reports focused on how oncologic outcomes are worse in these patients. We sought to investigate oncologic outcomes for these non-operated patients with rectal cancer after nCRT.

\section{Materials and Methods}

A total of 1,063 records of patients with rectal cancer who were treated with nCRT from January 2002 to December 2013 were retrospectively reviewed. We categorized patients into the non-operated group ( $n=77$ ), transanal local excision (TLE) group $(n=54)$, or total mesorectal excision (TME) group ( $n=932)$ and compared each group using propensity score matching.

\section{Results}

In the non-operated group, the most common reason for no surgery was patient refusal $(n=64)$. Eleven patients were considered to have achieve clinical complete response (cCR), which was an independent prognostic factor of progression-free survival $(p=0.045)$. In patients with disease progression in the non-operated group, the overall survival did not improved according to salvage treatments $(p=0.451)$. The non-operated group showed worse survivals compared to the TLE or TME group before and after matching $(p<0.001)$. This finding was also noted in the analysis of survival only in patients with cCR.

\section{Conclusion}

In this study, non-operated patients did not secure oncologic safety regardless of cCR after nCRT. Our results suggest that a non-operative management must be carefully considered even if cCR is achieved.
Key words

Rectal neoplasms, Neoadjuvant therapy, Chemoradiotherapy, Surgery 


\section{Introduction}

Rectal cancer is a common malignancy and almost 40,000 new cases develop annually in the United States [1,2]. Up to $70 \%$ of rectal cancer patients without metastasis present with locally advanced rectal cancer, defined as clinical stage T3-T4 and/or N1-N2 [3-5]. Rectal cancer treatment has changed in recent decades, resulting in improved outcomes [6]. Currently, neoadjuvant chemoradiotherapy (nCRT) followed by total mesorectal excision (TME) is the standard treatment for locally advanced rectal cancer [4,7]. nCRT can improve resectability, achieve better sphincter preservation, and reduce local recurrence [8]. Many studies have reported that nCRT with TME can improve survival outcome of rectal cancer patients [9-11].

Although TME is still the standard treatment for advanced rectal cancer, recent trends in minimally invasive treatments have led to an increase in local excision or "watch and wait" in patients with an excellent response to nCRT [5,6,12]. However, surgeons and oncologists occasionally meet patients who do not undergo surgery, regardless of tumor response for nCRT, for a number of reasons including advanced age, high comorbidities, patient refusal, or follow-up loss. These patients show poor oncologic outcomes compared to patients who receive optimal treatments, even if they achieve clinical complete response (cCR). Although a poor survival outcome is generally predicted for these patients, there have not been any reports on how oncologic outcomes are worse in this specific group. Thus, it is necessary to provide objective evidence of oncologic outcomes and inform expected disease progress. This analysis is also helpful for recommending the optimal treatment for these patients. In this study, we investigated the clinical features and oncologic outcomes of patients with rectal cancer who did not undergo surgery after nCRT for any reason.

\section{Materials and Methods}

\section{Patients}

A total of 1,063 records of patients with rectal cancer who were treated with nCRT from January 2002 to December 2013 at a tertiary university-based hospital were retrospectively reviewed. All patients had histologically-confirmed primary adenocarcinoma of the rectum located less than $10 \mathrm{~cm}$ from the anal verge. Patients were excluded if they did not complete nCRT or had recurrent disease, distant metastasis, synchronous malignancies, hereditary colorectal cancer, or pre- vious treatment for cancer. This study was approved by the Samsung Medical Center Institutional Review Board.

Patients were categorized into the non-operated group $(n=77)$, transanal local excision (TLE) group $(n=54)$, and TME group ( $\mathrm{n}=932$ ) according to treatment after nCRT (Fig. 1). Among 77 non-operated patients, 11 were considered cCR and 66 did not achieve cCR. The most common reason for not undergoing surgery was patient refusal $(n=64)$. High comorbidities $(n=7)$ and new metastasis $(n=5)$ were the next most common reasons. The remaining patient expired 8 days after completion of nCRT due to radiotherapy-induced toxicity. The patients in the TLE group did not undergo radical surgery due to patient refusal, pathologic CR (pCR), or comorbidities.

\section{Pretreatment evaluation and treatments}

Clinical TNM stage prior to nCRT was assessed radiologically using colonoscopy, endorectal ultrasonography (EUS), abdominopelvic computed tomography (APCT), pelvic magnetic resonance imaging (MRI), or positron emission tomography (PET). To achieve standardization of clinical staging, a single radiologist re-read the radiologic findings of the primary rectal lesion. All patients underwent preoperative radiotherapy consisting of 1.8-2.0 Gy daily fractions for a total dose of 44-54 Gy for 5 days per week. In our institution, a dose of 45 Gy was irradiated to patients until 2005. A total dose of 44 and 54 Gy were administered to patients in 20062008 and 2009-2010, respectively. After that date, a dose of $50.4 \mathrm{~Gy}$ was administered. Occasionally, one or two fractions may not be administered depending on the condition of patients. The mean dose of radiotherapy and standard deviation was $49.12 \pm 4.05 \mathrm{~Gy}$. Chemotherapy was administered concurrently with radiotherapy using either 5-fluorouracil (5-FU) or a capecitabine-based regimen. Among 1,063 patients, 899 received intravenous $5-\mathrm{FU}$ (425 $\mathrm{mg} / \mathrm{m}^{2} /$ day) and leucovorin ( $20 \mathrm{mg} / \mathrm{m}^{2} /$ day) for 5 days during the first and fifth weeks of radiotherapy. The others received oral capecitabine ( $825 \mathrm{mg} / \mathrm{m}^{2}$ / day) twice daily during radiotherapy.

cCR was assessed 6-8 weeks after the completion of nCRT with clinical and radiologic tools similar to those used in the baseline assessment of the tumor extent. In brief, cCR was defined according to the following criteria: absence of residual mass, ulceration, or significant irregularity of the rectal wall on digital rectal exam, colonoscopy, EUS, or APCT; absence of high signal intensity on diffusion-weighted imaging and low signal intensity of the apparent diffusion coefficient map in MRI; absence of significant focal discrete uptake on the rectal wall in PET; or no increase of post-treatment serum carcinoembryonic antigen (CEA) levels. Then, patients were recommended for radical surgery 6-10 weeks after com- 


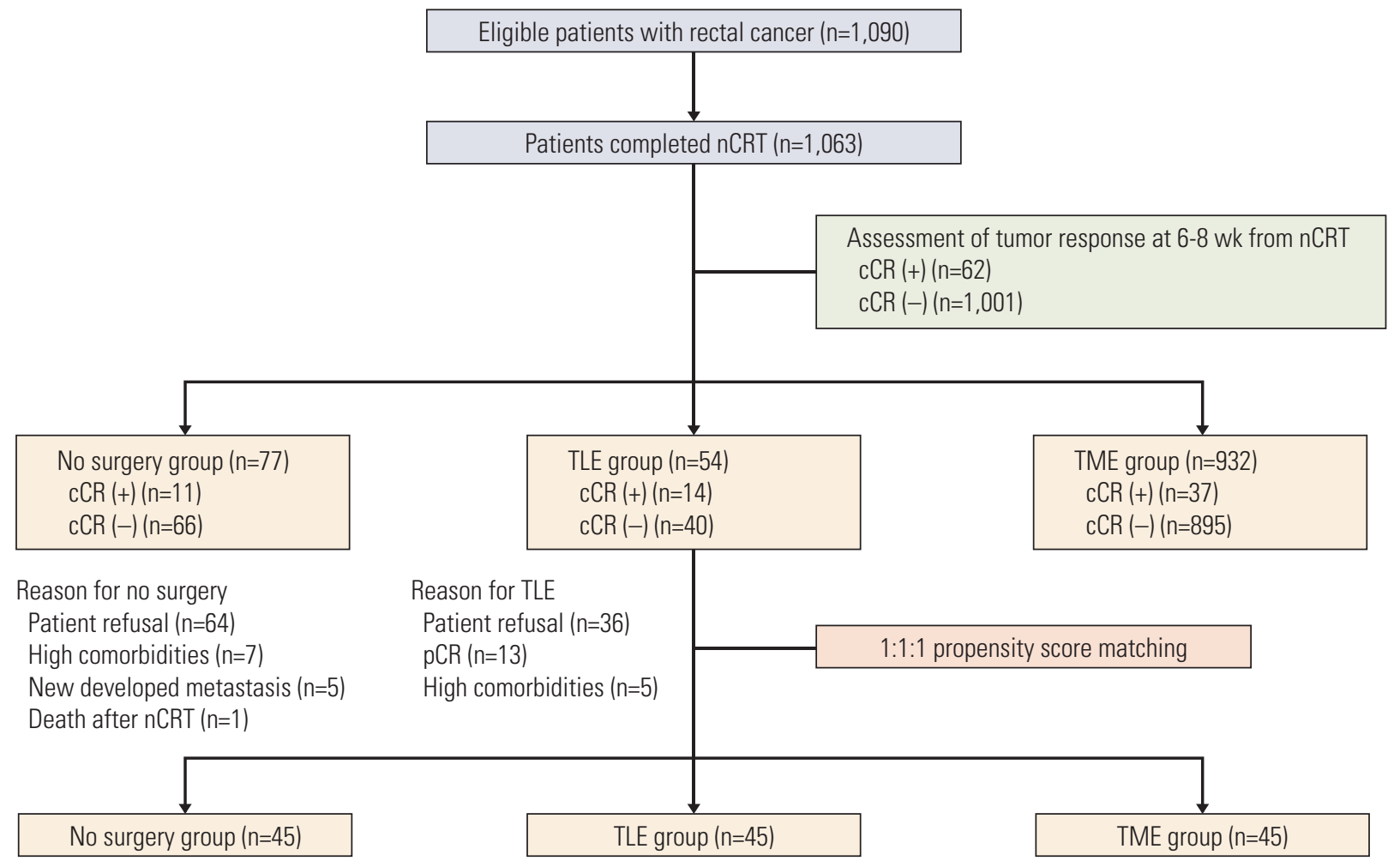

Fig. 1. Flow chart for patient selection. nCRT, neoadjuvant chemoradiotherapy; cCR, clinical complete response; TLE, transanal local excision; TME, total mesorectal excision; pCR, pathologic complete response.

pletion of nCRT. However, there were patients who received TLE or did not receive any surgery following nCRT for the previously stated reasons.

\section{Follow-up}

In patients who received surgery, postoperative surveillance visits happened every 3 months for the first 2 years and then every 6 months for up to 5 years. Most patients underwent physical examination and chest X-ray, and were evaluated for CEA level at each visit. APCT and chest computed tomography were performed every 6 months. Colonoscopy was performed at the first year and then biennially. Patients who did not receive surgery did not undergo routine surveillance. However, most of them visited every 3-6 months and underwent physical examination and chest $\mathrm{X}$-ray, and evaluation of serum CEA level. Among them, some patients underwent APCT every 6-12 months.

\section{Assessment of clinical outcomes}

Clinicopathologic features and survival outcomes were compared between groups. To analyze survival according to each group, we adjusted patient characteristics using propensity score matching to minimize confounding bias among the three groups. The patients were matched with a 1:1:1 ratio and 45 patients were included in each group (Fig. 1). We compared 2-year local progression-free survival (LPFS) and progression-free survival (PFS) because the median followup duration for progression was 19.4 months in the nonoperated group, 56.1 months in the TLE group and 52.3 months in the TME group. However, we analyzed 5-year overall survival (OS) because the median follow-up duration for death was 40.8 months in the non-operated group (TLE group, 60.1 months and TME group, 58.5 months). The primary endpoint of this study was survival outcome of the non-operated group, and the secondary endpoints were clinical features and prognostic factors in this group. 
Table 1. Patient characteristics before propensity score matching

\begin{tabular}{|c|c|c|c|c|}
\hline Characteristic & $\begin{array}{l}\text { Non-operated } \\
\text { group }(n=77)\end{array}$ & $\begin{array}{l}\text { TLE group } \\
(\mathrm{n}=54)\end{array}$ & $\begin{array}{l}\text { TME group } \\
\quad(n=932)\end{array}$ & p-value \\
\hline \multicolumn{5}{|l|}{ Age (yr) } \\
\hline$<65$ & $46(59.7)$ & $34(63.0)$ & $696(74.7)$ & 0.001 \\
\hline$\geq 65$ & $31(40.3)$ & $20(37.0)$ & $236(25.3)$ & \\
\hline \multicolumn{5}{|l|}{ Sex } \\
\hline Male & $50(64.9)$ & $33(61.1)$ & $628(67.4)$ & 0.468 \\
\hline Female & $27(35.1)$ & $21(38.9)$ & $304(32.6)$ & \\
\hline \multicolumn{5}{|l|}{ ASA score } \\
\hline 1 & $40(51.9)$ & $20(37.0)$ & $423(45.4)$ & 0.837 \\
\hline 2 & $32(41.6)$ & $32(59.3)$ & $485(52.0)$ & \\
\hline 3 & $4(5.2)$ & $2(3.7)$ & $23(2.5)$ & \\
\hline 4 & $1(1.3)$ & 0 & $1(0.1)$ & \\
\hline \multicolumn{5}{|l|}{ BMI $\left(\mathrm{kg} / \mathrm{m}^{2}\right)$} \\
\hline$<23$ & $41(53.2)$ & $19(35.2)$ & $363(38.9)$ & 0.038 \\
\hline$\geq 23$ & $36(46.8)$ & $35(64.8)$ & $569(61.1)$ & \\
\hline \multicolumn{5}{|c|}{ Pretreatment CEA $(\mathrm{ng} / \mathrm{mL})$} \\
\hline$<5$ & $52(67.5)$ & $51(94.4)$ & $880(94.4)$ & $<0.001$ \\
\hline$\geq 5$ & $25(32.5)$ & $3(5.6)$ & $52(5.6)$ & \\
\hline \multicolumn{5}{|l|}{ Cell type } \\
\hline $\mathrm{WD} / \mathrm{MD}$ & $71(92.2)$ & $50(92.6)$ & $852(91.4)$ & 0.747 \\
\hline PD/MUC/SRC & $6(7.8)$ & $4(7.4)$ & $80(8.6)$ & \\
\hline \multicolumn{5}{|c|}{ Pretreatment clinical T stage } \\
\hline $\mathrm{T} 1-2$ & $14(18.2)$ & $28(51.9)$ & $89(9.5)$ & $<0.001$ \\
\hline $\mathrm{T} 3$ & $53(68.8)$ & $26(48.1)$ & 717 (76.9) & \\
\hline $\mathrm{T} 4$ & $10(13.0)$ & 0 & $126(13.5)$ & \\
\hline \multicolumn{5}{|c|}{ Pretreatment clinical N stage } \\
\hline Negative & $16(20.8)$ & $37(68.5)$ & $212(22.7)$ & 0.027 \\
\hline Positive & $61(79.2)$ & $17(31.5)$ & $720(77.3)$ & \\
\hline \multicolumn{5}{|l|}{ Clinical CR } \\
\hline No & $66(85.7)$ & $40(74.1)$ & $895(96.0)$ & $<0.001$ \\
\hline Yes & $11(14.3)$ & $14(25.9)$ & $37(4.0)$ & \\
\hline \multicolumn{5}{|l|}{ Pathologic stage } \\
\hline Pathologic CR & - & $24(44.4)$ & $164(17.6)$ & $<0.001$ \\
\hline 0 & - & $7(13.0)$ & $17(1.8)$ & \\
\hline I & - & $21(38.9)$ & $246(26.4)$ & \\
\hline II & - & $2(3.7)$ & $234(29.1)$ & \\
\hline III & - & 0 & $271(29.1)$ & \\
\hline \multicolumn{5}{|l|}{ Lymphatic invasion } \\
\hline No & - & $28(51.8)$ & $611(65.6)$ & $<0.001$ \\
\hline Yes & - & $1(1.9)$ & $108(11.6)$ & \\
\hline Unknown & - & $25(46.3)$ & $213(22.8)$ & \\
\hline \multicolumn{5}{|l|}{ Vascular invasion } \\
\hline No & - & $16(29.6)$ & $565(60.6)$ & $<0.001$ \\
\hline Yes & - & 0 & $67(7.2)$ & \\
\hline Unknown & - & $38(70.4)$ & $300(32.2)$ & \\
\hline
\end{tabular}

(Continued to the next page) 
Table 1. Continued

\begin{tabular}{|c|c|c|c|c|}
\hline Characteristic & $\begin{array}{l}\text { Non-operated } \\
\text { group }(n=77)\end{array}$ & $\begin{array}{l}\text { TLE group } \\
(\mathrm{n}=54)\end{array}$ & $\begin{array}{l}\text { TME group } \\
(\mathrm{n}=932)\end{array}$ & p-value \\
\hline \multicolumn{5}{|c|}{ Perineural invasion } \\
\hline No & - & $16(29.6)$ & $573(61.5)$ & $<0.001$ \\
\hline Yes & - & 0 & $93(10.0)$ & \\
\hline Unknown & - & $38(70.4)$ & $266(28.5)$ & \\
\hline
\end{tabular}

Values are presented as number (\%). TLE, transanal excision; TME, total mesorectal excision; ASA, American Society of Anesthesiologists; BMI, body mass index; CEA, carcinoembryonic antigen; WD, well differentiated; MD, moderately differentiated; PD, poorly differentiated; MUC, mucinous carcinoma; SRC, signet ring cell carcinoma; CR, complete response.



Fig. 2. Clinical course of patients who did not receive surgery after neoadjuvant chemoradiotherapy. Tx, treatment.

\section{Statistical analysis}

Statistical analysis was performed using SPSS for Windows ver. 20.0 (IBM SPSS Statistics, IBM Corp., Armonk, NY). Categorical variables were compared using the chisquare test and Fisher exact probability. Propensity score matching was performed to adjust for patient characteristics, and then survival rates were analyzed using the KaplanMeier method and log-rank test. Multivariate analyses for prognostic factors were performed in a Cox proportional hazards model with stepwise inclusion of variables. $p$-values were derived from two-tailed tests and $p<0.05$ was considered statistically significant.

\section{Results}

\section{Clinical features of the non-operated group}

Among a total of 1,063 patients, 77 were in the non-operated group and the most common reason they did not undergo surgery was patient refusal $(n=64)$. In the nonoperated group, the median age was 61.0 years (range, 33 to 82 years) and 50 patients were male. Of these 77 patients, 11 were considered cCR and 66 did not achieve cCR (Table 1).

In survival analysis, 2-year LPFS and PFS were $49.0 \%$ and $43.0 \%$, respectively. The median follow-up duration for progression was 19.4 months. The 5-year OS was 58.3\% and the 


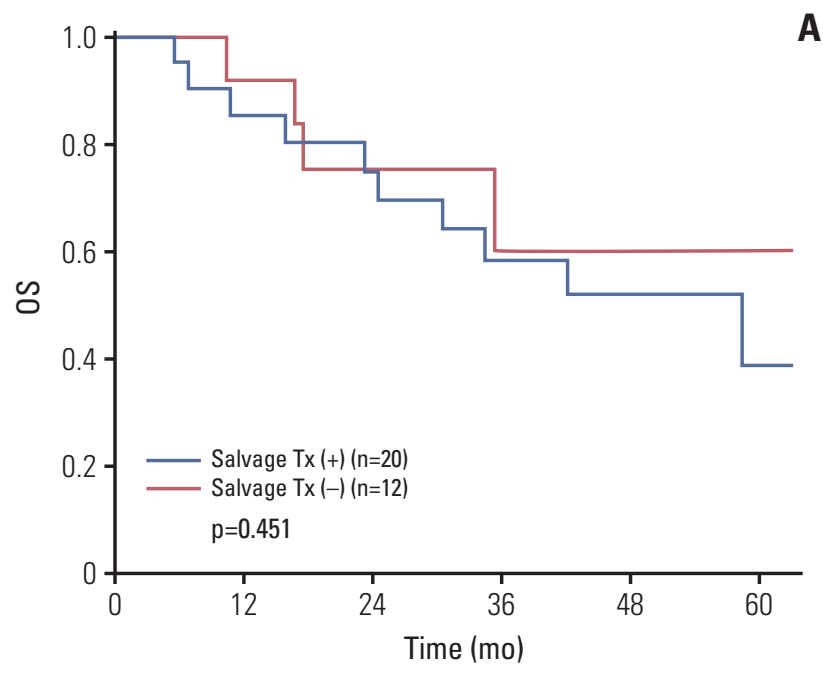

A
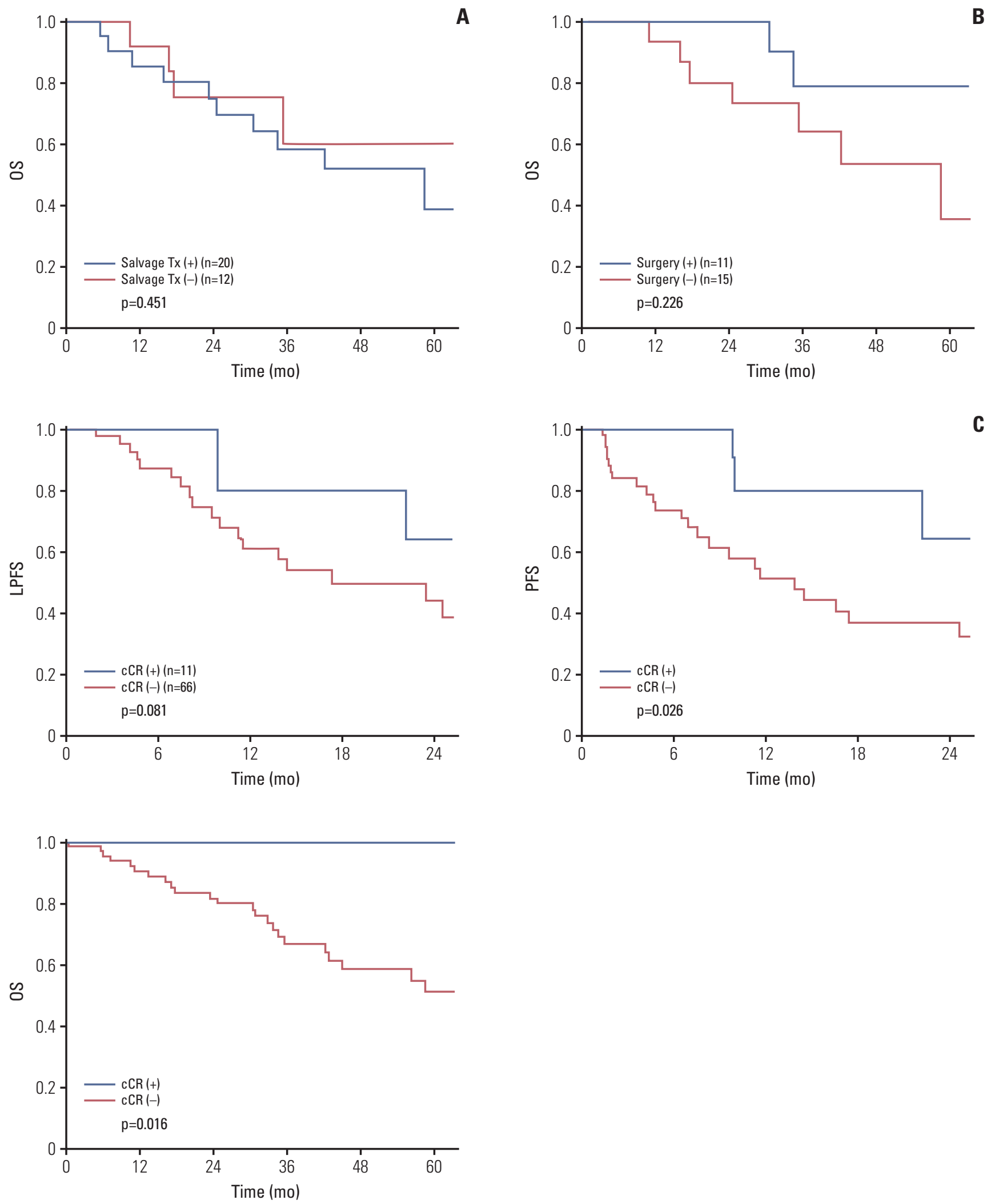

Fig. 3. Kaplan-Meir plots of non-operated group. (A) Overall survival (OS) according to salvage treatment (Tx) in progressed patients ( $n=32)$. (B) OS according to salvage surgery in locally progressed patients $(n=26)$. (C) Local progression-free survival (LPFS), progression-free survival (PFS), and OS according to clinical complete response (cCR). 
Table 2. Multivariate analysis of prognostic factor for overall patients of non-operated group

\begin{tabular}{|c|c|c|c|c|}
\hline & \multicolumn{2}{|c|}{ PFS } & \multicolumn{2}{|c|}{ OS } \\
\hline & p-value & OR $(95 \% \mathrm{CI})$ & p-value & OR $(95 \% \mathrm{CI})$ \\
\hline \multicolumn{5}{|l|}{ Cell type } \\
\hline $\mathrm{PD} / \mathrm{MUC} / \mathrm{SRC}$ vs. WD/MD & 0.002 & $8.779(2.154-35.788)$ & 0.021 & $3.229(1.189-8.766)$ \\
\hline \multicolumn{5}{|l|}{ Pretreatment clinical N stage } \\
\hline Node $(+)$ vs. node $(-)$ & & & 0.100 & $2.795(0.820-9.523)$ \\
\hline \multicolumn{5}{|l|}{ Clinical complete response } \\
\hline Yes vs. no & 0.045 & $0.337(0.116-0.977)$ & - & - \\
\hline
\end{tabular}

PFS, progression-free survival; OS, overall survival; OR, odds ratio; CI, confidence interval; PD, poorly differentiated; MUC, mucinous carcinoma; SRC, signet ring cell carcinoma; WD, well differentiated; MD, moderately differentiated.

Table 3. Patient characteristics after propensity score matching

\begin{tabular}{|c|c|c|c|c|}
\hline Characteristic & $\begin{array}{c}\text { Non-operated } \\
\text { group }(n=45)\end{array}$ & $\begin{array}{l}\text { TLE group } \\
(n=45)\end{array}$ & $\begin{array}{l}\text { TME group } \\
\quad(n=45)\end{array}$ & p-value \\
\hline \multicolumn{5}{|l|}{ Age (yr) } \\
\hline$<65$ & $26(57.8)$ & $30(66.7)$ & $32(71.1)$ & 0.186 \\
\hline$\geq 65$ & $19(42.2)$ & $15(33.3)$ & $13(28.9)$ & \\
\hline \multicolumn{5}{|l|}{ Sex } \\
\hline Male & $28(62.2)$ & $29(64.4)$ & $25(55.6)$ & 0.521 \\
\hline Female & $17(37.8)$ & $16(35.6)$ & $20(44.4)$ & \\
\hline \multicolumn{5}{|l|}{ ASA } \\
\hline 1 & $22(48.9)$ & $17(37.8)$ & $21(46.7)$ & 0.583 \\
\hline 2 & $19(42.2)$ & $26(57.8)$ & $24(53.3)$ & \\
\hline 3 & $4(8.9)$ & $2(4.4)$ & 0 & \\
\hline \multicolumn{5}{|l|}{ BMI $\left(\mathrm{kg} / \mathrm{m}^{2}\right)$} \\
\hline$<23$ & $24(53.3)$ & $15(33.3)$ & $18(40.0)$ & 0.202 \\
\hline$\geq 23$ & $21(46.7)$ & $30(66.7)$ & $27(60.0)$ & \\
\hline \multicolumn{5}{|c|}{ Pretreatment CEA (ng/mL) } \\
\hline$<5$ & $34(75.6)$ & $36(80.0)$ & $32(71.1)$ & 0.625 \\
\hline$\geq 5$ & $11(24.4)$ & $9(20.0)$ & $13(28.9)$ & \\
\hline \multicolumn{5}{|l|}{ Cell type } \\
\hline $\mathrm{WD} / \mathrm{MD}$ & $43(95.6)$ & $41(91.1)$ & $42(93.3)$ & 0.674 \\
\hline PD/MUC/SRC & $2(4.4)$ & $4(8.9)$ & $3(6.7)$ & \\
\hline \multicolumn{5}{|c|}{ Pretreatment clinical T stage } \\
\hline $\mathrm{T} 1-2$ & $13(28.9)$ & $21(46.7)$ & $14(31.1)$ & 0.826 \\
\hline $\mathrm{T} 3$ & $32(71.1)$ & $24(53.3)$ & $31(68.9)$ & \\
\hline \multicolumn{5}{|c|}{ Pretreatment clinical $\mathrm{N}$ stage } \\
\hline Negative & $13(28.9)$ & $29(64.4)$ & $19(42.2)$ & 0.205 \\
\hline Positive & $32(71.1)$ & $16(35.6)$ & $26(57.8)$ & \\
\hline \multicolumn{5}{|c|}{ Clinical complete response } \\
\hline No & $38(84.4)$ & $34(75.6)$ & $41(91.1)$ & 0.134 \\
\hline Yes & $7(15.6)$ & $11(24.4)$ & $4(8.9)$ & \\
\hline
\end{tabular}

Values are presented as number (\%). TLE, transanal excision; TME, total mesorectal excision; ASA, American Society of Anesthesiologists; BMI, body mass index; CEA, carcinoembryonic antigen; WD, well differentiated; MD, moderately differentiated; PD, poorly differentiated; MUC, mucinous carcinoma; SRC, signet ring cell carcinoma. 

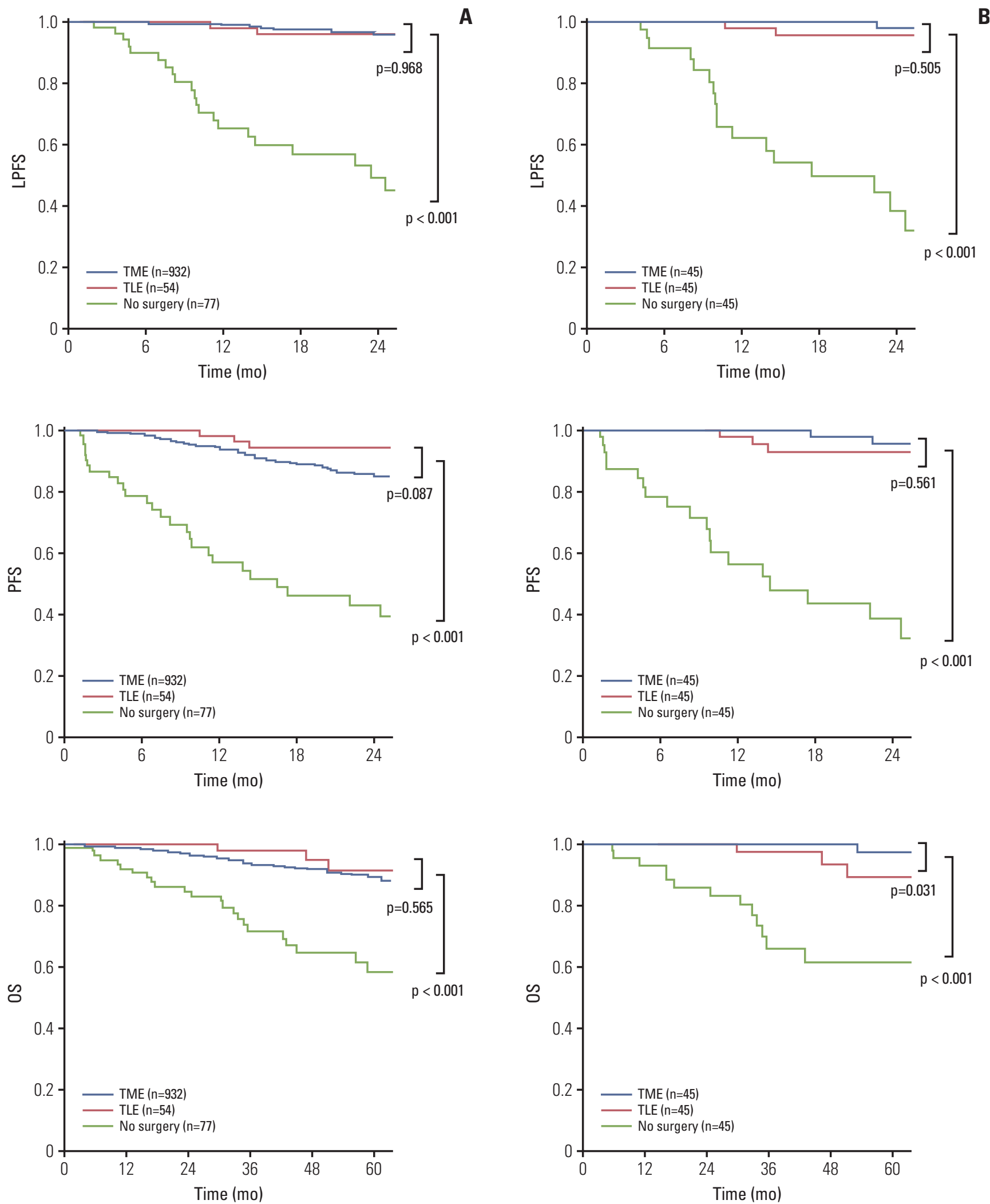

Fig. 4. Kaplan-Meir plots of local progression-free survival (LPFS), progression-free survival (PFS), and overall survival (OS) according to surgery group. (A) Before matching $(\mathrm{n}=1,063)$. (B) After matching $(\mathrm{n}=135)$. (Continued to the next page) 

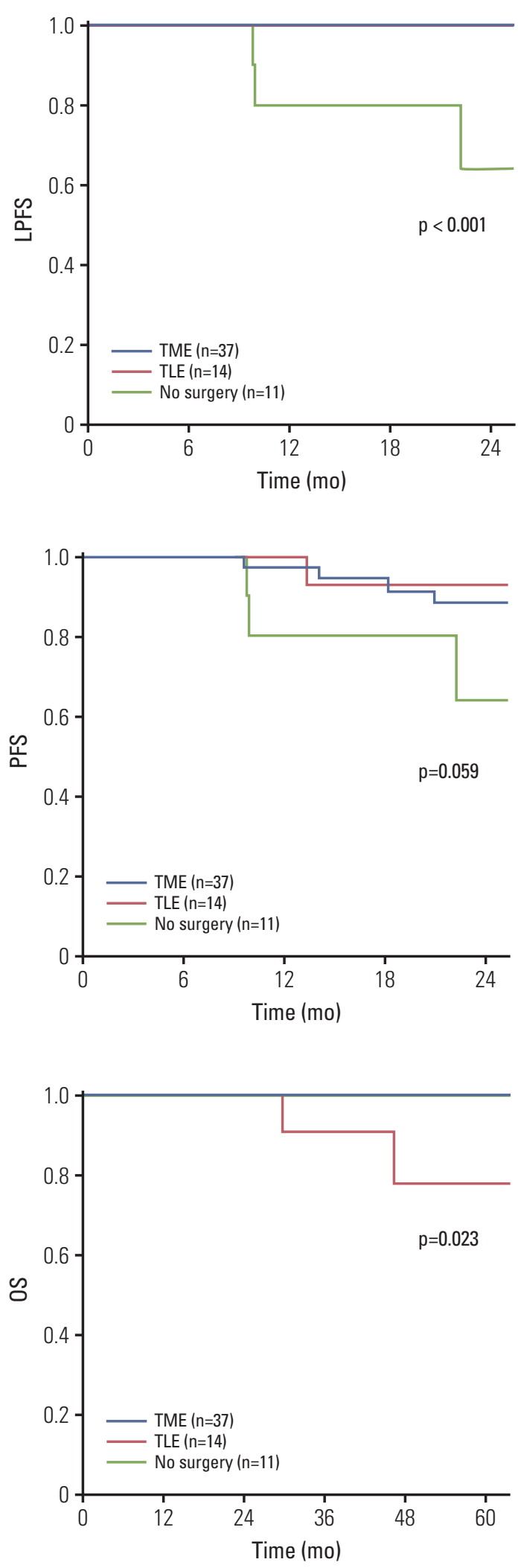

Fig. 4. (Continued from the previous page) (C) Survival outcomes of patients with clinical complete response $(n=62)$. TME, total mesorectal excision; TLE, transanal local excision. median follow-up duration for death was 40.8 months. During the follow-up period, 32 patients experienced local or systemic progression and 25 expired. In 32 patients with disease progression, 20 received salvage treatments such as surgery or chemotherapy (Fig. 2). To investigate clinical features of progressed patients of the non-operated surgery group, further analyses of survival were performed. In overall progressed patients $(n=32)$, OS was not different according to salvage treatments for their progression $(\mathrm{p}=0.451)$ (Fig. 3A). In locally progressed patients $(n=26)$, similarly, there was no significant difference in OS depending on whether they had received salvage surgery for progressed lesions $(p=0.226)$ (Fig. 3B). Among 11 patients who underwent salvage surgery, in addition, eight underwent abdominoperineal resection, two underwent palliative T-colostomy, and one underwent palliative TLE. In the survival analysis according to $\mathrm{CCR}, 11$ patients with $\mathrm{CCR}$ showed better survival outcomes than those without $\mathrm{cCR}(\mathrm{n}=66)(\mathrm{p}=0.081, \mathrm{p}=0.026$, and $\mathrm{p}=0.016$ in LPFS, PFS, and OS, respectively) (Fig. 3C).

For non-operated patients, the Cox proportional hazard model was performed to identify prognostic factors for survival. Factors that were significant or nearly significant $(p<0.100)$ in the univariate analysis were included in the multivariate analysis. In the multivariate analysis, non-cCR was an independent poor prognostic factor of PFS ( $p=0.045$ ). In addition, poor histology was a poor prognostic factor of PFS and OS ( $\mathrm{p}=0.002$ and $\mathrm{p}=0.021$, respectively) (Table 2 ).

\section{Patient characteristics according to surgery}

To evaluate clinical features of the non-operated group, clinical characteristics were compared to the TLE and TME group. Among a total of 1,063 patients, 77 were non-operated patients, and 54 and 932 were in the TLE and TME group, respectively. Older patients were more common in the nonoperated group compared to the TLE and TME groups. Pretreatment clinical $\mathrm{T}$ and $\mathrm{N}$ stage were more advanced in the non-operated group and TME group compared to the TLE group. In addition, the CCR rate was the highest in TLE group (Table 1). Therefore, we adjusted the patients with 1:1:1 ratio using propensity score matching for variables described in Table 3 and 45 patients were included in each group. After matching, we determined that the patients in each group were balanced for all variables (Table 3).

\section{Survival outcomes according to surgery}

To investigate the survival outcomes of the non-operated group, we analyzed the 2-year LPFS, PFS, and 5-year OS for the non-operated, TLE and TME groups. Before matching, as expected, the non-operated patients showed significantly decreased LPFS, PFS, and OS rates compared to the patients 
Table 4. Multivariate analysis of prognostic factor after propensity score matching $(\mathrm{n}=135)$

\begin{tabular}{|c|c|c|c|c|}
\hline & \multicolumn{2}{|c|}{ PFS } & \multicolumn{2}{|c|}{ OS } \\
\hline & p-value & OR $(95 \% \mathrm{CI})$ & p-value & OR $(95 \% \mathrm{CI})$ \\
\hline Group & $<0.001$ & & 0.001 & \\
\hline TLE vs. no surgery & $<0.001$ & $0.118(0.048-0.293)$ & 0.026 & $0.268(0.084-0.854)$ \\
\hline TME vs. no surgery & $<0.001$ & $0.061(0.023-0.162)$ & 0.001 & $0.032(0.004-0.254)$ \\
\hline \multicolumn{5}{|l|}{ BMI $\left(\mathrm{kg} / \mathrm{m}^{2}\right)$} \\
\hline$\geq 23$ vs. $<23$ & 0.331 & $0.704(0.347-1.428)$ & & \\
\hline \multicolumn{5}{|c|}{ Pretreatment clinical N stage } \\
\hline Node $(+)$ vs. node $(-)$ & 0.144 & $1.742(0.827-3.671)$ & 0.063 & $2.646(0.945-8.092)$ \\
\hline
\end{tabular}

PFS, progression-free survival; OS, overall survival; OR, odds ratio; CI, confidence interval; TLE, transanal excision; TME, total mesorectal excision; BMI, body mass index.

of other groups ( $\mathrm{p}<0.001$, all) (Fig. 4A). After matching, we still determined that the survival rates of the non-operated group were the lowest ( $\mathrm{p}<0.001$, all) (Fig. 4B). Subsequently, we investigated survival outcomes of patients with $\mathrm{CCR}$ between three groups ( $\mathrm{n}=11,14$, and 37 in non-operated group, TLE group, and TME group, respectively), and observed that the patients of the non-operated group showed worse LPFS and PFS than those of the other two groups $(p<0.001$ and $p=0.059$, respectively). However, no patient with cCR in the non-operated group expired (Fig. 4C).

For matched patients, Cox proportional hazard model was performed to identify prognostic factors for survival. Factors that were significant or nearly significant $(p<0.100)$ in univariate analysis were included in the multivariate analysis. Results of multivariate analysis revealed that no surgery was the only independent poor prognostic factor of PFS and OS in rectal cancer patients who received nCRT ( $p<0.001$ and $\mathrm{p}=0.001$, respectively) (Table 4 ).

\section{Discussion}

In this study, we investigated disease progress of rectal cancer patients who did not receive surgery regardless of tumor response for nCRT. For both overall and matched patients, the non-operated patient group showed worse survival outcomes compared to the patients who underwent TME or TLE after nCRT. This finding was also noted in the analysis of survival only in patients with CCR.

Recently, there has been growing evidence of the oncologic feasibility of non-operative management for the patients with cCR after nCRT in rectal cancer. Studies from the HabrGama group reported good local control rates and long-term survival in patients with cCR after nCRT [13-15]. Another multicenter study reported that there was no significant difference in survival between the non-operated group with CCR and the operated group after nCRT [16]. In addition, other study groups reported similar results [5,17]. In contrast, several studies have suggested lack of oncologic safety with non-operative management in the patients with CCR [18-20]. However, these studies have focused only the patients with CCR and did not provide objective data for cases without cCR. In the current study, we evaluated the oncologic outcomes of non-operated patients with CCR and without cCR. We observed that non-operated patients showed worse survival outcomes than those underwent surgery regardless their tumor response. In the cases with cCR, local progression was the highest in the non-operated group compared to the TME and TLE group. These results suggest that a nonoperative management must be carefully considered even if cCR is achieved. The patients with cCR expect good survival, but they may still harbor residual disease. Concordance between cCR and $\mathrm{pCR}$ is a major concern in these cases. There is not yet a consensus on the "watch and wait" policy in the field of rectal cancer and our data did not support this policy. Clinical trials to suggest an appropriate approach for these patients are warranted.

Currently, local excision or non-operative management is considered to be an optional therapeutic strategy for patients showing excellent response after nCRT [12,13,21-23]. The controversy surrounding these patients persists, however, and there have not been any reports on how oncologic outcomes are worse in the patients who did not undergo surgical resection despite non-cCR after nCRT. Clinicians have met these non-operated patients who refused surgery due to improved symptoms after nCRT, high comorbidities, old age, fear of permanent stoma, etc. In our group, 64 of the 77 patients who did not undergo surgery refused surgery after 
nCRT. In these cases, clinicians should recommend standard treatments. However, there are few objective data showing a clear clinical course. In our study, the non-operated patients showed significantly worse survival outcomes than patients who underwent surgery and there was no survival gain from salvage treatments after progression. In addition, 10 of 11 patients who underwent salvage surgery had permanent stoma despite being considered appropriate for sphincter preservation at presentation. The TLE group showed significantly better survival than the non-operated group although it was worse than the TME group. Thus, it is better to recommend local excision rather than no surgery when patients refuse surgery or are at high risk for radical surgery.

There were some limitations to our study. This study was retrospectively performed at a single institution. Thus, the follow-up duration for progression of the non-operated group was shorter than that of the other two groups, so we could not analyze long-term PFS. However, we analyzed long-term OS by following whether patients were alive or expired. Another limitation was the lack of equal distribution among the groups. Thus, we performed propensity score matched analysis to minimize confounding bias between groups. In addition, the decision to do not undergo surgery was mostly driven by patient refusal, favoring under-investigation during their disease course. This can lead to low early detection rates of progression and poor oncologic outcomes. Despite these limitations, to our knowledge, this is the first study focusing on patients who did not undergo surgery despite not achieving CCR after completion of nCRT. Although a poor survival outcome is generally predicted for these patients, objective data on this topic is needed. In addition, we included a large number of patients who did not receive any surgery, underwent local excision, or underwent radical surgery regardless of their tumor response.

In this study, we showed bad disease progress in nonoperated patients regardless of tumor response after nCRT and no survival gain from salvage treatments after progression in these patients. Our results suggest that a non-operative management must be carefully considered even if $\mathrm{cCR}$ is achieved.

\section{Conflicts of Interest}

Conflict of interest relevant to this article was not reported.

\section{Acknowledgments}

This study was supported by Basic Science Research Program through the National Research Foundation of Korea (NRF) funded by the Ministry of Education, Science and Technology (grant number 2015R1A1A1A05001160).

\section{References}

1. Fokas E, Liersch T, Fietkau R, Hohenberger W, Beissbarth T, Hess $\mathrm{C}$, et al. Tumor regression grading after preoperative chemoradiotherapy for locally advanced rectal carcinoma revisited: updated results of the $\mathrm{CAO} / \mathrm{ARO} / \mathrm{AIO}-94$ trial. J Clin Oncol. 2014;32:1554-62.

2. Arredondo J, Baixauli J, Rodriguez J, Beorlegui C, Arbea L, Zozaya G, et al. Patterns and management of distant failure in locally advanced rectal cancer: a cohort study. Clin Transl Oncol. 2016;18:909-14.

3. Huang MY, Wu CH, Huang CM, Chung FY, Huang CW, Tsai HL, et al. DPYD, TYMS, TYMP, TK1, and TK2 genetic expressions as response markers in locally advanced rectal cancer patients treated with fluoropyrimidine-based chemoradiotherapy. Biomed Res Int. 2013;2013:931028.

4. Wallin U, Rothenberger D, Lowry A, Luepker R, Mellgren A. CEA: a predictor for pathologic complete response after neoadjuvant therapy for rectal cancer. Dis Colon Rectum. 2013;56:859-68.

5. Lai CL, Lai MJ, Wu CC, Jao SW, Hsiao CW. Rectal cancer with complete clinical response after neoadjuvant chemoradiotherapy, surgery, or "watch and wait". Int J Colorectal Dis. 2016;31:
413-9.

6. Beets GL, Figueiredo NL, Habr-Gama A, van de Velde CJ. A new paradigm for rectal cancer: Organ preservation: Introducing the International Watch \& Wait Database (IWWD). Eur J Surg Oncol. 2015;41:1562-4.

7. Oh BY, Park YA, Huh JW, Cho YB, Yun SH, Lee WY, et al. Metformin enhances the response to radiotherapy in diabetic patients with rectal cancer. J Cancer Res Clin Oncol. 2016;142: 1377-85.

8. Hur H, Kim NK, Min BS, Baik SH, Lee KY, Koom WS, et al. Can a biomarker-based scoring system predict pathologic complete response after preoperative chemoradiotherapy for rectal cancer? Dis Colon Rectum. 2014;57:592-601.

9. Gollins S, Sebag-Montefiore D. Neoadjuvant treatment strategies for locally advanced rectal cancer. Clin Oncol (R Coll Radiol). 2016;28:146-51.

10. Lee SH, Lee KC, Choi JH, Oh JH, Baek JH, Park SH, et al. Chemoradiotherapy followed by surgery in rectal cancer: improved local control using a moderately high pelvic radiation dose. Jpn J Clin Oncol. 2008;38:112-21.

11. Yeh $\mathrm{CH}$, Chen MF, Lai $\mathrm{CH}$, Huang WS, Lee SP, Chen WC. 
Comparison of treatment results between surgery alone, preoperative short-course radiotherapy, or long-course concurrent chemoradiotherapy in locally advanced rectal cancer. Int J Clin Oncol. 2012;17:482-90.

12. Hingorani M, Hartley JE, Greenman J, Macfie J. Avoiding radical surgery after pre-operative chemoradiotherapy: a possible therapeutic option in rectal cancer? Acta Oncol. 2012;51: 275-84.

13. Habr-Gama A, Sabbaga J, Gama-Rodrigues J, Sao Juliao GP, Proscurshim I, Bailao Aguilar P, et al. Watch and wait approach following extended neoadjuvant chemoradiation for distal rectal cancer: are we getting closer to anal cancer management? Dis Colon Rectum. 2013;56:1109-17.

14. Smith FM, Rao C, Oliva Perez R, Bujko K, Athanasiou T, Habr-Gama A, et al. Avoiding radical surgery improves early survival in elderly patients with rectal cancer, demonstrating complete clinical response after neoadjuvant therapy: results of a decision-analytic model. Dis Colon Rectum. 2015;58: 159-71.

15. Habr-Gama A, Gama-Rodrigues J, Sao Juliao GP, Proscurshim I, Sabbagh C, Lynn PB, et al. Local recurrence after complete clinical response and watch and wait in rectal cancer after neoadjuvant chemoradiation: impact of salvage therapy on local disease control. Int J Radiat Oncol Biol Phys. 2014;88: 822-8.

16. Renehan AG, Malcomson L, Emsley R, Gollins S, Maw A, Myint AS, et al. Watch-and-wait approach versus surgical resection after chemoradiotherapy for patients with rectal cancer (the OnCoRe project): a propensity-score matched cohort analysis. Lancet Oncol. 2016;17:174-83.

17. Li J, Liu H, Yin J, Liu S, Hu J, Du F, et al. Wait-and-see or radical surgery for rectal cancer patients with a clinical complete response after neoadjuvant chemoradiotherapy: a cohort study. Oncotarget. 2015;6:42354-61.

18. Hughes R, Harrison M, Glynne-Jones R. Could a wait and see policy be justified in T3/4 rectal cancers after chemo-radiotherapy? Acta Oncol. 2010;49:378-81.

19. dos Santos LV, dos Anjos Jacome AA, Carcano FM, da Silveira Nogueira Lima JP, Serrano SV. Watch and wait policy remains experimental for the management of rectal cancer. Colorectal Dis. 2010;12:833.

20. Glynne-Jones R, Hughes R. Critical appraisal of the 'wait and see' approach in rectal cancer for clinical complete responders after chemoradiation. Br J Surg. 2012;99:897-909.

21. Yu CS, Yun HR, Shin EJ, Lee KY, Kim NK, Lim SB, et al. Local excision after neoadjuvant chemoradiation therapy in advanced rectal cancer: a national multicenter analysis. Am J Surg. 2013;206:482-7.

22. Noh JM, Park W, Kim JS, Koom WS, Kim JH, Choi DH, et al. Outcome of local excision following preoperative chemoradiotherapy for clinically T2 distal rectal cancer: a multicenter retrospective study (KROG 12-06). Cancer Res Treat. 2014;46: 243-9.

23. Lee NK, Kim DY, Kim SY, Oh JH, Park W, Choi DH, et al. Clinical outcomes of local excision following preoperative chemoradiotherapy for locally advanced rectal cancer. Cancer Res Treat. 2014;46:158-64. 